\title{
Models for Nuclear Smuggling Interdiction
}

\author{
David P. Morton ${ }^{1}$ \\ Graduate Program in Operations Research \\ 1 University Station, C2200 \\ The University of Texas at Austin \\ Austin, TX 78712-0292, USA \\ morton@mail .utexas.edu \\ 512-471-4104 (voice) \\ 512-471-8717 (fax) \\ Feng Pan and Kevin J. Saeger \\ D-6, Risk Analysis \& Decision Support System \\ Los Alamos National Laboratory \\ Mail Stop F604 \\ Los Alamos, NM 87545, USA \\ fpan@lanl.gov, saeger@lanl.gov
}

September 2005

\footnotetext{
${ }^{1}$ Corresponding Author
} 


\title{
Models for Nuclear Smuggling Interdiction
}

\author{
David P. Morton \\ Graduate Program in Operations Research \\ The University of Texas at Austin \\ Austin, TX 78712, USA \\ morton@mail .utexas .edu \\ Feng Pan and Kevin J. Saeger \\ Risk Analysis \& Decision Support System \\ Los Alamos National Laboratory \\ Los Alamos, NM 87545, USA \\ fpan@lanl.gov, saeger@lanl.gov
}

September 2005

\begin{abstract}
We describe two stochastic network interdiction models for thwarting nuclear smuggling. In the first model, the smuggler travels through a transportation network on a path that maximizes the probability of evading detection, and the interdictor installs radiation sensors to minimize that evasion probability. The problem is stochastic because the smuggler's origin-destination pair is known only through a probability distribution at the time when the sensors are installed. In this model, the smuggler knows the locations of all sensors and the interdictor and the smuggler "agree" on key network parameters, namely the probabilities the smuggler will be detected while traversing the arcs of the transportation network. Our second model differs in that the interdictor and smuggler can have differing perceptions of these network parameters. This model captures the case in which the smuggler is aware of only a subset of the sensor locations. For both models, we develop the important special case in which the sensors can only be installed at border crossings of a single country so that the resulting model is defined on a bipartite network. In this special case, a class of valid inequalities reduces the computation time for the identical-perceptions model.
\end{abstract}

\section{Introduction}

We develop stochastic network interdiction models designed to locate radiation sensors, which detect gamma and neutron emissions from nuclear material, at critical border crossings in the Former Soviet Union (FSU). The goal is to locate the sensors on an underlying transportation network to minimize the probability of a successful smuggling attempt. Our work supports the Second Line of Defense (SLD) program of the US Department of Energy. 
Smuggling of nuclear material, equipment, and technology has become a greater threat to international security since the dissolution of the Soviet Union. In the early 1990s, Russia inherited roughly 600-850 metric tons of highly-enriched uranium and plutonium, enough material to make over 50,000 explosive devices $[12,18]$. The "first line of defense" concerns nuclear material protection, control and accountability (MPC\&A). In short, this involves securing and inventorying nuclear material at its storage sites in both civilian and defense facilities.

An International Atomic Energy Agency (IAEA) database includes 540 incidents of trafficking of nuclear and radioactive material from 1993-2003 that have been confirmed by a country's government [23]. 205 of these involved nuclear material and 17 involved weaponsgrade uranium or plutonium. With sophisticated technology, non-weapons-grade nuclear material can be processed to obtain weapons-grade material. With minimal technology it, or more widely-available radioactive material, can be used with conventional explosives to build a radiological dispersal device, i.e., a dirty bomb. The majority of the incidents involved smugglers seeking to sell the illicit material. Weapons-grade material has been seized by authorities in Russia, Germany, the Czech Republic, Lithuania, Bulgaria, Kyrgyzstan, Georgia, Greece and France, and in the majority of the cases the material was traced to have originated in Russia or other parts of the FSU [23]. Some incidents involved kilograms of material. Some others involving smaller quantities actually represented samples of stolen material or material at risk of being stolen. This clearly points to the vulnerability of Russia's first line of defense. US efforts to assist the FSU in improving Russia's first line of defense are ongoing [16]. These MPC\&A efforts are critically important but by themselves, insufficient. An accurate inventory of the nuclear material that existed in Russia at the beginning of the 1990s seems impossible.

The SLD program seeks to reduce the risk of illicit trafficking of nuclear material through airports, seaports and border crossings in Russia and other key transit states, with the program's initial efforts in the FSU $[4,32]$. The first SLD sensor installation was at Moscow's Sheremetyevo International Airport in September of 1998. Such sensor installations have two purposes: (i) to deter potential theft and smuggling of nuclear material and (ii) to detect and therefore prevent actual smuggling attempts.

In this paper, we describe two types of stochastic network interdiction models that can be used to select the sites to install sensors to minimize the probability a smuggler can travel through a transportation network undetected. Our two basic models are distinguished with respect to whether the interdictor and smuggler have the same or differing perceptions 
of key network parameters. Our first model, in which the smuggler and interdictor have identical perceptions of the network, has been developed in collaboration with the Los Alamos National Laboratory SLD team and has been implemented for decision-support for the SLD program. Our second model in which the interdictor and smuggler can have differing perceptions is an important extension. The primary emphasis in this paper is on modeling, as opposed to solution techniques and computation. Of course, modeling choices affect our ability to solve these problems, and so important parts of the development are devoted to precisely these issues. Furthermore, we describe, and motivate from a modeling perspective, a class of valid inequalities that strengthen our simplest model. Finally, in developing our basic model, we provide an outline of some of the techniques that have been successfully employed to obtain tractable network interdiction models in settings beyond the specific models of this paper.

While there are earlier references (e.g., [38]), the study of network interdiction in operations research began in earnest in the 1970s. During the Vietnam War, deterministic mathematical programs to disrupt flow of enemy troops and supplies were developed $[20,30]$. The problem of maximizing an adversary's shortest path is considered in $[17,21]$. A closely-related problem concerns maximizing the longest path in an adversary's PERT network $[10,35]$. When these are linear programs (LPs), the interdictor can continuously increase the length of an arc, subject to a budget constraint. A discrete version of maximizing the shortest path removes an interdicted arc from the network, and when the budget constraint is simply a cardinality constraint, this is called the $k$-most-vital arcs problem $[5,13,29]$. Generalizations of the $k$-most-vital arcs problem are considered in [25]. The interdiction problem of removing arcs to minimize flow in an adversary's maximum-flow network is considered in $[38,40]$. See [37] for game-theoretic approaches to related network interdiction problems, [11] for an interdiction model on a minimum-cost-flow network, and [24] for interdiction models of more general systems.

The above interdiction models are deterministic in the following senses. First, the arc lengths in the shortest-path and PERT problems, and the arc capacities in the maximumflow problem, are known with certainty. Second, when increasing the length of an arc in the former problems or when removing or decreasing the capacity of an arc in the latter problem, these modifications are deterministic, i.e., with certainty. The work of [40] on maximumflow network interdiction is generalized in [14] to allow for both random arc capacities and interdiction successes. An interdiction model with uncertain network topology is developed in [22]. A stochastic interdiction model in which the adversary's response is modeled via a 
Markov decision process is considered in [3].

The remainder of this paper is organized as follows. In Section 2, we formulate our basic model, which we label SNIP, for stochastic network interdiction problem, as a bilevel stochastic mixed-integer program. This model exhibits a "min-max" structure, which does not lend itself to computation and so we formulate an equivalent stochastic linear mixedinteger program (MIP) that can be solved, e.g., by commercial branch-and-bound solvers for integer programming. We then turn our attention in Section 3 to an important special case of SNIP that arose in our work on the SLD program, in which sensors can only be installed at border crossings of a single country, namely Russia. We show the associated MIP can be simplified in this special case. The resulting model is called BiSNIP, for bipartite stochastic network interdiction problem, because it may be viewed as an interdiction problem on a bipartite network. Section 4 generalizes SNIP and BiSNIP to models we call PSNIP and BiPSNIP, respectively. Here, the addition of "P" to the SNIP and BiSNIP labels indicates that these are models in which the interdictor and the evader differ in their perceptions of the network. Our emphasis here is on the simpler BiPSNIP case. In Section 5, we describe a class of valid inequalities, that we call step inequalities, to tighten the MIP formulation of BiSNIP, and we present computational results when using these inequalities. We conclude the paper in Section 6.

\section{SNIP on a General Network}

We model two adversaries, an interdictor and an evader (we will use the terms "evader" and "smuggler" interchangeably), and an underlying directed network $G(N, A)$ on which the evader travels. In the deterministic version of our model, the evader starts at a source node $s \in N$ and wishes to reach a terminal node $t \in N$. The model is deterministic in that this origin-destination pair is known. The probability that the evader can traverse arc $(i, j) \in A$ undetected is $p_{i j}$ if the interdictor has not installed a sensor on arc $(i, j)$, and this probability is $q_{i j}<p_{i j}$ if the interdictor has. An evader can be caught by indigenous law enforcement without radiation detection equipment, and so $p_{i j}<1$. The events of the evader being detected on distinct arcs are assumed to be mutually independent. The evader chooses an $s$ - $t$ path to maximize the probability of traversing the network without being detected. With limited resources, the interdictor must select arcs on which to install sensors in order to minimize this evasion probability.

Our stochastic network interdiction problem (SNIP) differs from the above description 
only in that the identity of the evader is unknown when the interdictor installs the sensors. In our basic SNIP model, an evader's identify is uniquely specified by an origin-destination pair, $(s, t)$, which is assumed to be governed by a known probability mass function, $p^{\omega}=$ $P\left\{(s, t)=\left(s^{\omega}, t^{\omega}\right)\right\}, \omega \in \Omega$. The probability evader $\omega$ traverses the network undetected is then a sum of (conditional) evasion probabilities, each weighted by $p^{\omega}$, over the population of possible evaders indexed by $\omega \in \Omega$. While our basic model equates evader $\omega$ 's identity with an origin-destination pair, straightforward extensions we describe below allow this identify to involve further distinguishing characteristics.

The timing of decisions and realizations is as follows: First, the interdictor installs sensors on a subset of the network's arcs. Then, a random origin-destination pair is revealed and evader $\omega$ selects an $s^{\omega}-t^{\omega}$ path to maximize the probability of avoiding detection. The evader selects this path with full knowledge of the sensor locations and evasion probabilities.

As indicated above, the model represents sensor installations as occurring on arcs. In reality, detection equipment (e.g., in the form of radiation sensor portals) is installed at transportation choke points such as airports, seaports, and international border crossings for automobiles, railroads and pedestrians. We capture this in our model by splitting the node associated with such a location into two nodes with an arc representing travel through the checkpoint.

In SNIP, we assume smugglers are aware of the sensor locations. The initial installation of SLD equipment in Moscow in 1998 was accompanied by a ribbon-cutting ceremony [15], and subsequent installations were also reported in the news. The reason for this publicity is that completely sealing Russia's 12,500 miles of borders is impractical in today's environment. As a result, in addition to catching nuclear smugglers, the SLD program seeks to deter would-be smugglers. That said, as the program expands beyond Russia these policies may change. So, in Section 4 we consider variants of SNIP in which the smuggler has a perception of the network parameters $p_{i j}$ and $q_{i j}$ that may differ from that of the interdictor and in which the smuggler is aware of only a subset of the sensor locations. The notation used in formulating SNIP is summarized below, followed by the formulation.

\section{Network and Sets}

$G(N, A)$ directed network with nodes $N$ and $\operatorname{arcs} A$

$F S(i) \quad$ set of arcs leaving node $i$

$R S(i) \quad$ set of arcs entering node $i$

$A D \subset A$ arcs on which sensors may be placed 


\section{Data}

$b$

total budget for installing sensors

$c_{i j}$

cost of installing a sensor on arc $(i, j) \in A D$

$p_{i j}$

probability evader can traverse $(i, j)$ undetected with no sensor installed

$q_{i j}$

probability evader can traverse $(i, j)$ undetected with a sensor installed;

$q_{i j}<p_{i j}$

\section{Random Elements}

$\omega \in \Omega \quad$ sample point and sample space, indexing evader's identity

$\left(s^{\omega}, t^{\omega}\right) \quad$ realization of evader's origin-destination pair

$p^{\omega} \quad$ probability mass function

\section{Interdictor's Decision Variables}

$x_{i j} \quad 1$ if a sensor is installed on $\operatorname{arc}(i, j)$ and 0 otherwise

\section{Evader's Decision Variables}

$y_{i j} \quad$ positive only if evader traverses $(i, j)$ and no sensor is installed

$z_{i j} \quad$ positive only if evader traverses $(i, j)$ and a sensor is installed

\section{Boundary Conditions}

$x_{i j}, z_{i j} \equiv 0 \quad(i, j) \notin A D$

\section{Formulation}

$$
\min _{x \in X} \sum_{\omega \in \Omega} p^{\omega} h\left(x,\left(s^{\omega}, t^{\omega}\right)\right)
$$

where $X=\left\{x: \sum_{(i, j) \in A D} c_{i j} x_{i j} \leq b, x_{i j} \in\{0,1\},(i, j) \in A D\right\}$, and where $h\left(x,\left(s^{\omega}, t^{\omega}\right)\right)$ is the optimal value of

$$
\begin{array}{ll}
\max _{y, z} & y_{t^{\omega}} \\
\text { s.t. } & \sum_{\left(s^{\omega}, j\right) \in F S\left(s^{\omega}\right)}\left(y_{s^{\omega} j}+z_{s^{\omega} j}\right)=1: \pi_{s^{\omega}} \\
& \sum_{(i, j) \in F S(i)}\left(y_{i j}+z_{i j}\right)=\sum_{(j, i) \in R S(i)}\left(p_{j i} y_{j i}+q_{j i} z_{j i}\right), i \in N \backslash\left\{s^{\omega}, t^{\omega}\right\}: \pi_{i} \\
& y_{t^{\omega}}=\sum_{\left(j, t^{\omega}\right) \in R S\left(t^{\omega}\right)}\left(p_{j t^{\omega}} y_{j t^{\omega}}+q_{j t^{\omega}} z_{j t^{\omega}}\right): \pi_{t^{\omega}} \\
& 0 \leq y_{i j} \leq 1-x_{i j}, \quad(i, j) \in A: \lambda_{i j} \\
& 0 \leq z_{i j}, \quad(i, j) \in A D .
\end{array}
$$

The optimal value of (2) is the conditional probability that the smuggler avoids detection, given $\left(s^{\omega}, t^{\omega}\right)$. The objective function in (1) is the expected value of this conditional evasion probability, where the expectation is taken over all possible origin-destination pairs. Dual variables $\pi_{i}, i \in N$, and $\lambda_{i j},(i, j) \in A$, are shown in (2) with their corresponding constraints. 
Each network link on which a sensor can be placed is modeled as two arcs in parallel. If a sensor is installed, i.e., $x_{i j}=1$, then flow may occur only on the "sensor" arc, through $z_{i j}$. Otherwise flow can occur on the "no sensor" arc, via $y_{i j}$. Flow on $\operatorname{arc}(i, j)$ is multiplied by that arc's gain (either $p_{i j}$ or $q_{i j}$ ). So, if $P_{s^{\omega}, t^{\omega}}$ is a path from $s^{\omega}$ to $t^{\omega}$ then

$$
y_{t^{\omega}}=\prod_{(i, j) \in P_{s} \omega, t^{\omega}}\left[p_{i j}\left(1-x_{i j}\right)+q_{i j} x_{i j}\right]
$$

is the probability that evader $\omega$ can travel $P_{s^{\omega}, t^{\omega}}$ without being detected. The evader's subproblem finds a path $P_{s^{\omega}, t^{\omega}}$ maximizing $y_{t^{\omega}}$ by forcing one unit of flow out of $s^{\omega}$ in $(2 \mathrm{~b})$, enforcing flow conservation at all intermediate nodes in (2c), defining the flow that reaches $t^{\omega}$ as $y_{t^{\omega}}$ in $(2 \mathrm{~d})$ and maximizing that value in (2a). Flow is forced on the appropriate arc, and incurs the associated gain (actually, loss), by the interdictor's decision variable $x_{i j}$ in (2e). Note that an upper bound of $x_{i j}$ in (2f) is not necessary since $q_{i j}<p_{i j}$ implies the smuggler will not use $z_{i j}$ in place of $y_{i j}$ when $x_{i j}=0$.

When locating sensors, the interdictor knows: (i) the network topology, (ii) the indigenous detection probability on each arc, (iii) the detection probability given the presence of a sensor, (iv) the budget constraint, (v) the probability distribution governing the random $(s, t)$ pair, and (vi) the method by which the evader will select a path. After $\left(s^{\omega}, t^{\omega}\right)$ is revealed, evader $\omega$ selects an $s^{\omega}-t^{\omega}$ path that maximizes the evasion probability, knowing (i), (ii) and (iii) as well as the sensor locations.

In the model's current form, $p_{i j}$ and $q_{i j}$ are identical for all possible smugglers, and scenario $\omega$ simply specifies the smuggler's origin-destination pair, $\left(s^{\omega}, t^{\omega}\right)$. However, the above formulation can directly accommodate the case when these are replaced by $p_{i j}^{\omega}$ and $q_{i j}^{\omega}$. In this setting, the smuggler's identity, $\omega$, specifies the origin-destination pair, $\left(s^{\omega}, t^{\omega}\right)$, and the evasion probabilities, $p_{i j}^{\omega}$ and $q_{i j}^{\omega}$. Here, the evasion probabilities could differ for smugglers having the same origin-destination pair because of differences in the nature of the material being smuggled and how the material is packaged (these affect a sensor's ability to detect the material), and could also include other traits of the smuggler, which could affect the likelihood of detection via indigenous law enforcement. For notational simplicity, we will not label $p_{i j}$ and $q_{i j}$ with $\omega$.

SNIP with $h$ defined in (2) is a bilevel stochastic mixed-integer program. In bilevel programs (e.g., $[7,8,26]$ ) each player has an objective function, and these can differ because the players' motives differ. In our case, the objective function is the same for both players, but the interdictor is minimizing that function and the evader is maximizing it. The problem is formulated with a nested "min-max" structure, which is not amenable to solution through 
standard optimization algorithms. There are at least three approaches one might take to obtain a tractable optimization model from a problem like SNIP, and we label these decomposition, duality and reformulation.

- Decomposition: The nested min-max structure is not a difficulty if one can employ an outer-approximation cutting-plane algorithm like Kelley's cutting-plane method [27], Benders' decomposition $[9,19]$, or the L-Shaped method [36, 39]. Unfortunately, $h\left(x,\left(s^{\omega}, t^{\omega}\right)\right)$, defined by (2), is a maximization linear program with $x$ appearing on the right-hand side of $(2 \mathrm{e})$. This implies that $h\left(x,\left(s^{\omega}, t^{\omega}\right)\right)$, and hence $E h\left(x,\left(s^{\omega}, t^{\omega}\right)\right)$, is a concave function over the convex hull of $X$. This does not bode well for employing the above cutting-plane schemes. We note that Laporte and Louveaux [28] have developed variants of the L-Shaped method that are valid for nonconvex $E h\left(x,\left(s^{\omega}, t^{\omega}\right)\right)$ but we regard this approach as one of last resort if our other approaches fail because they require cutting planes that are tight at a specific (binary) value of $x=\hat{x}$ and drop to an a priori lower bound at all other (binary) values of $x \in X$.

- Duality: Another general approach is to take the dual of the inner maximization problem and then to formulate a single model in which we simultaneously optimize over the interdictor's decision variables and the smuggler's (dual) decision variables. We assume the network $G$ is such that an $s^{\omega}-t^{\omega}$ path exists for all $\omega \in \Omega$. So, (2) is feasible and has a finite optimal solution for all $\omega \in \Omega$. Hence, an equivalent expression of $h\left(x,\left(s^{\omega}, t^{\omega}\right)\right)$ is available via linear programming duality, i.e., using the dual variables as indicated in (2) we have

$$
\begin{array}{rlrl}
h\left(x,\left(s^{\omega}, t^{\omega}\right)\right)=\min _{\pi, \lambda} & \pi_{s^{\omega}}+\sum_{(i, j) \in A D}\left(1-x_{i j}\right) \lambda_{i j} & \\
\text { s.t. } & \pi_{i}-p_{i j} \pi_{j} \geq 0, & (i, j) \in A \backslash A D \\
& \pi_{i}-p_{i j} \pi_{j}+\lambda_{i j} \geq 0, & (i, j) \in A D \\
& \pi_{i}-q_{i j} \pi_{j} \geq 0, & (i, j) \in A D \\
& \lambda_{i j} \geq 0, & & (i, j) \in A D \\
& \pi_{t^{\omega}}=1 . &
\end{array}
$$

With the expression of $h\left(x,\left(s^{\omega}, t^{\omega}\right)\right)$ in $(4)$, we have a nested "min-min" formulation and can now form a single optimization problem in which we simultaneously minimize over $x$ and $\left(\pi^{\omega}, \lambda^{\omega}\right), \omega \in \Omega$. However, the associated objective function includes nonlinear terms 
between $x$ and $\lambda^{\omega}$ so we first linearize these:

$$
\begin{aligned}
& \min _{\pi, \lambda, \beta} \pi_{s} \omega+\sum_{(i, j) \in A D} \beta_{i j} \\
& \text { s.t. } \quad \pi_{i}-p_{i j} \pi_{j} \geq 0, \quad(i, j) \in A \backslash A D \\
& \pi_{i}-p_{i j} \pi_{j}+\lambda_{i j} \geq 0, \quad(i, j) \in A D \\
& \pi_{i}-q_{i j} \pi_{j} \geq 0, \quad(i, j) \in A D \\
& \beta_{i j}-\lambda_{i j} \geq-x_{i j}, \quad(i, j) \in A D \\
& \lambda_{i j}, \beta_{i j} \geq 0, \quad(i, j) \in A D \\
& \pi_{t^{\omega}}=1 \text {. }
\end{aligned}
$$

Constraints (5a) and (5b) can replaced by $\pi_{i}-p_{i j} \pi_{j}+\beta_{i j} \geq-x_{i j},(i, j) \in A D$, eliminating variables $\lambda_{i j},(i, j) \in A D$, in the process. Next, it is clear that we can take $\beta_{i j}=0$ in an optimal solution and the model simplifies to

$$
\begin{array}{cll}
h\left(x,\left(s^{\omega}, t^{\omega}\right)\right)=\min _{\pi} & \pi_{s^{\omega}} & \\
\text { s.t. } & \pi_{i}-p_{i j} \pi_{j} \geq 0, & (i, j) \in A \backslash A D \\
& \pi_{i}-p_{i j} \pi_{j} \geq-x_{i j}, \quad(i, j) \in A D \\
& \pi_{i}-q_{i j} \pi_{j} \geq 0, & (i, j) \in A D \\
& \pi_{t^{\omega}}=1 .
\end{array}
$$

Model (6) leads us to a single large-scale linear MIP in which we minimize over $x$ and $\pi^{\omega}$, $\omega \in \Omega$. Our above reasoning in linearizing the nonlinear terms in (4)'s objective function mirrors that of Wood's [40] application of the duality approach to the interdiction problem on a maximum-flow network. Below, we arrive at (6) through another, arguably simpler approach. However, the duality approach is important in interdiction modeling because it is one way to arrive at a tractable model, and in some cases, such as the shortest-path interdiction model of [17], the duality approach is the simplest in that it does not give rise to the types of nonlinear terms we have seen above.

- Reformulation: In [34], we showed a minor variant of the following result: $h\left(x,\left(s^{\omega}, t^{\omega}\right)\right)$ as defined in (2) may be equivalently formulated as

$$
\begin{gathered}
h\left(x,\left(s^{\omega}, t^{\omega}\right)\right)=\max _{y \geq 0, z \geq 0} y_{t^{\omega}}-\sum_{(i, j) \in A D} x_{i j} y_{i j} \\
\text { s.t. } \quad(2 \mathrm{~b})-(2 \mathrm{~d}) .
\end{gathered}
$$

Model (7) differs from (2) in that the upper bound in (2e) has been removed and a penalty term is now included in the objective function. The intuition behind this reformulation 
is that while we now allow flow on $y_{i j}$ even if a sensor is installed on $(i, j)$, i.e., $x_{i j}=1$, in that case, we subtract the associated flow in the objective function. The validity of the reformulation is based on an exact penalty result from [31]. A proof is also given above in our application of the duality approach, where we argued $h\left(x,\left(s^{\omega}, t^{\omega}\right)\right)$ is given by (6) because (7) is (6)'s dual. As mentioned above, the duality approach was applied to maximum-flow network interdiction in [40]. The reformulation approach we consider here was applied to (stochastic versions of) the same maximum-flow problem in [14]. The following theorem tightens the reformulation in (7).

Theorem 1 Assume that $G$ has an $s^{\omega}-t^{\omega}$ path $\forall \omega \in \Omega, 0 \leq p_{i j} \leq 1,(i, j) \in A$, and $0 \leq q_{i j}<p_{i j},(i, j) \in A D$. Then, for all $x \in X$ and $\omega \in \Omega, h\left(x,\left(s^{\omega}, t^{\omega}\right)\right)$ is the optimal value of the following linear program

$$
\begin{array}{cll}
\min _{\pi} & \pi_{s^{\omega}} & \\
\text { s.t. } & \pi_{i}-p_{i j} \pi_{j} \geq 0, & (i, j) \in A \backslash A D \\
& \pi_{i}-p_{i j} \pi_{j} \geq-\left(p_{i j}-q_{i j}\right) x_{i j}, & (i, j) \in A D \\
& \pi_{i}-q_{i j} \pi_{j} \geq 0, & (i, j) \in A D \\
& \pi_{t^{\omega}}=1 . &
\end{array}
$$

Proof: Model (7) has dual (8) except that constraints (8a) are instead

$$
\pi_{i}-p_{i j} \pi_{j} \geq-x_{i j}, \quad(i, j) \in A D .
$$

If $x_{i j}=0$ then (8a) and (9) are equivalent. On the other hand, if $x_{i j}=1$ then

$$
\pi_{i}-q_{i j} \pi_{j}=\pi_{i}-p_{i j} \pi_{j}+\left(p_{i j}-q_{i j}\right) \pi_{j} x_{i j} \leq \pi_{i}-p_{i j} \pi_{j}+\left(p_{i j}-q_{i j}\right) x_{i j},
$$

where the inequality holds since $p_{i j}>q_{i j}$ and $0 \leq \pi_{j} \leq 1$ in any optimal solution of (7)'s dual. Thus, when $x_{i j}=1$ constraint ( $\left.8 \mathrm{a}\right)$ is redundant given $(8 \mathrm{~b})$.

The value of Theorem 1 is that SNIP can be expressed as the following large-scale MIP

$$
\begin{array}{lll}
\min _{x, \pi} & \sum_{\omega \in \Omega} p^{\omega} \pi_{s}^{\omega} & \\
\text { s.t. } & x \in X & \\
& \pi_{i}^{\omega}-p_{i j} \pi_{j}^{\omega} \geq 0, & (i, j) \in A \backslash A D, \omega \in \Omega \\
& \pi_{i}^{\omega}-p_{i j} \pi_{j}^{\omega}+\left(p_{i j}-q_{i j}\right) x_{i j} \geq 0, & (i, j) \in A D, \omega \in \Omega \\
& \pi_{i}^{\omega}-q_{i j} \pi_{j}^{\omega} \geq 0, & (i, j) \in A D, \omega \in \Omega \\
& \pi_{t^{\omega}}=1, & \omega \in \Omega .
\end{array}
$$


In summary, the goal of all three techniques (decomposition, duality and reformulation) is to achieve a computationally-tractable optimization model. In various settings each technique has proven useful for attaining this goal. In our case, the duality and reformulation approaches lead to the same model. That said, the reformulation approach may be more attractive in that it provides an intuitive modeling-based argument for the formulation and avoids the need to carryout the types of transformations we performed from (4) to (6), which may not always be so straightforward.

We can now attempt to solve the large-scale MIP (10) via commercially-available integer programming software. Interestingly, the reformulations (6), (7) and (8) also make our problem amenable to solution by the L-Shaped decomposition method. As earlier noted, $h\left(x,\left(s^{\omega}, t^{\omega}\right)\right)$ as defined by $(2)$ is concave over the convex hull of $X$. However, $h\left(x,\left(s^{\omega}, t^{\omega}\right)\right)$ as defined by (8) is convex over the convex hull of $X$ because it is a minimization linear program with $x$ in the right-hand side. (Having $h$ be both convex and concave over the convex hull of $X$ is possible because the optimal values of these linear programs are only ensured to be equal when $x$ takes on binary values.) The L-Shaped method with a MIP master program is an attractive solution approach when the number of scenarios $|\Omega|$ is large.

To close this section, we comment on one other modeling approach that could prove valuable in certain settings. In model (2), the smuggler is solving what is called the maximum-reliability path problem, and it is well known that by taking logarithms of the objective function as expressed in (3) the model can be solved as a shortest-path problem (see, e.g., [1, exercise 4.39]). When $|\Omega|=1$ this approach leads to a linear MIP formulation by first taking logarithms and then using the type of "duality" approach outlined above. However, when $|\Omega|>1$ one cannot simply take logarithms of each $h$ term in $\sum_{\omega \in \Omega} p^{\omega} h\left(x,\left(s^{\omega}, t^{\omega}\right)\right)$ and obtain an equivalent objective function. Instead, one can write $\sum_{\omega \in \Omega} p^{\omega} \exp \left\{\ln \left[h\left(x,\left(s^{\omega}, t^{\omega}\right)\right)\right]\right\}$. This leads to a nonlinear MIP, and so we prefer the above approach which begins with the generalized network flow formulation in (2) and leads to a linear MIP. Next, we turn to an important special case of SNIP on a bipartite network.

\section{BiSNIP: SNIP on a Bipartite Network}

The most pressing problem in our initial SLD work concerned smuggling of nuclear material out of Russia. In this special case of SNIP, potential sensor locations are restricted to customs checkpoints. With origins $\left(s^{\omega}\right)$ located inside Russia and destinations $\left(t^{\omega}\right)$ located outside Russia, the key to simplifying the formulation is that on each $s^{\omega}-t^{\omega}$ path there is 
exactly one customs checkpoint arc on which the smuggler could encounter a sensor.

Let $\mathcal{P}^{\omega}$ denote the set of all paths for origin-destination pair $\left(s^{\omega}, t^{\omega}\right)$. In BiSNIP we assume that each path in $\mathcal{P}^{\omega}$ contains exactly one arc in $A D$, i.e., each path has exactly one arc that can receive a sensor. Let $A D^{\omega}=\left\{(i, j):(i, j) \in A D,(i, j) \in \mathcal{P}^{\omega}\right\}$ be all such checkpoint arcs for $\omega \in \Omega$. For each $\omega$, we compute the value of the maximum-reliability path from $s^{\omega}$ to the tail of each checkpoint arc, and the value of the maximum-reliability path from the head of each checkpoint arc to $t^{\omega}$. (As indicated in the previous section, this can be done efficiently.) Let the product of these two probabilities be $\gamma_{k}^{\omega}, k=(i, j) \in A D^{\omega}$. The probability the smuggler can travel from $s^{\omega}$ to $t^{\omega}$ via $k$ undetected is $\gamma_{k}^{\omega} p_{k}$ if no sensor is installed at $k$, and this probability is $\gamma_{k}^{\omega} q_{k}$ if a sensor is installed at $k$. Smuggler $\omega$ 's path-selection decision is now reduced to the decision of choosing a checkpoint $k$ through which to travel, and that checkpoint is found by solving

$$
h\left(x,\left(s^{\omega}, t^{\omega}\right)\right)=\max _{k \in A D^{\omega}}\left\{\gamma_{k}^{\omega} p_{k}\left(1-x_{k}\right), \gamma_{k}^{\omega} q_{k} x_{k}\right\} .
$$

As a result, we can express BiSNIP as the following stochastic MIP

$$
\begin{array}{ll}
\min _{x, \theta} & \sum_{\omega \in \Omega} p^{\omega} \theta^{\omega} \\
\text { s.t. } & x \in X \\
& \theta^{\omega} \geq \gamma_{k}^{\omega} p_{k}\left(1-x_{k}\right), \quad k \in A D^{\omega}, \omega \in \Omega \\
& \theta^{\omega} \geq \gamma_{k}^{\omega} q_{k} x_{k}, \quad k \in A D^{\omega}, \omega \in \Omega .
\end{array}
$$

The bipartite network on which BiSNIP is defined has node sets $\Omega$ and $A D=\cup_{\omega \in \Omega} A D^{\omega}$, and $\operatorname{arcs}(\omega, k)$ link each origin-destination pair $\left(s^{\omega}, t^{\omega}\right), \omega \in \Omega$, with its possible intermediate checkpoints, $k \in A D^{\omega}$. Figure 1 illustrates the transformation from the underlying transportation network to the corresponding bipartite network.

Since $p_{k}>q_{k}$ and $x_{k}$ is binary, we can replace the right-hand side of (11b) with $\gamma_{k}^{\omega} q_{k}$, and hence, replace (11b) with the simple lower bound $\theta^{\omega} \geq \bar{q}^{\omega} \equiv \max _{k \in A D^{\omega}} \gamma_{k}^{\omega} q_{k}, \omega \in \Omega$. Defining $\bar{\theta}^{\omega}=\theta^{\omega}-\bar{q}^{\omega}$, we transform (11) to a model in which $\bar{\theta}^{\omega}$ has simple lower bounds of zero,

$$
\begin{array}{ll}
\min _{x, \bar{\theta}} & \sum_{\omega \in \Omega} p^{\omega} \bar{\theta}^{\omega} \\
\text { s.t. } & x \in X \\
& \bar{\theta}^{\omega} \geq r_{k}^{\omega}\left(1-x_{k}\right), k \in A D^{\omega}, \omega \in \Omega,
\end{array}
$$

where $r_{k}^{\omega}=\left(\gamma_{k}^{\omega} p_{k}-\bar{q}^{\omega}\right)^{+}$and where $(\cdot)^{+}=\max (\cdot, 0)$. The simplified model (12) is equivalent to (11) in that they have the same set of optimal solutions for locating the sensors, 


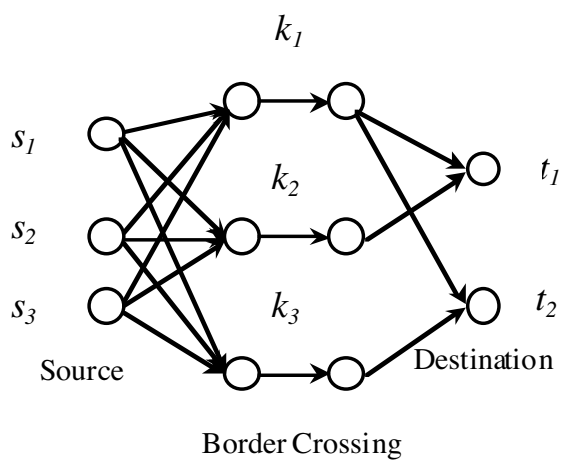

(a)

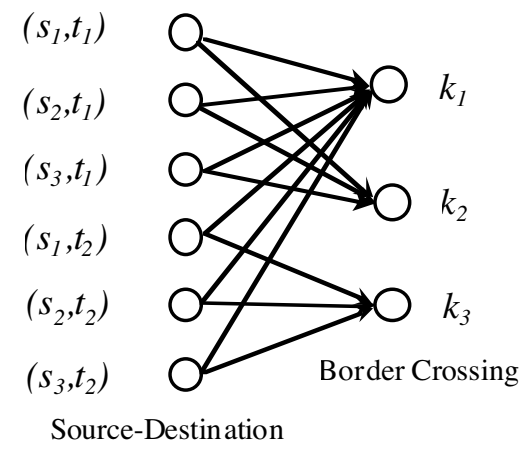

(b)

Figure 1: (a) The figure shows the result of preprocessing the underlying transportation network to a network in which one set of arcs connects sources with the tails of bordering-crossing arcs and another set of arcs connects the heads of bordering-crossing arcs with destinations. (b) The figure shows the final step of the transformation which yields a bipartite network structure connecting each scenario's $\left(s^{\omega}, t^{\omega}\right)$ pair with its possible border crossings $k \in A D^{\omega}$.

but their objective functions differ by a constant. We can view this as a transformation to a model in which the radiation sensors are perfectly reliable, i.e., (12) has the form of (11) with $q_{k}=0$.

\section{4 (Bi)PSNIP: (Bi)SNIP with Differing Perceptions}

In the previous two sections we developed SNIP on a general network and then specialized it to a bipartite network. In SNIP the interdictor and the evader agree upon, i.e., have the same perception of, the network topology $G(N, A)$, the indigenous detection probability on each arc, $p_{i j},(i, j) \in A$, and the detection probability given the presence of a sensor, $q_{i j}$, $(i, j) \in A D$. This section generalizes SNIP to a model we call PSNIP to handle the case in which the evader and the interdictor differ in their perceptions of these network parameters (The network topology remains agreed upon.) and then focus on the special case of PSNIP in which we are restricted to locating sensors on arcs leaving a single country, i.e., model BiPSNIP.

Static Stackelberg programs and other bilevel programs deal with asymmetries in players' objective functions and information. In a sense, stochastic network interdiction captures an asymmetry in that the interdictor does not know which evader will appear from a population of possible evaders. That said, there has been little work in the interdiction literature on handling other types of asymmetries. One notable exception is recent work on 
shortest-path network interdiction [2].

We redefine and extend the notation of Section 2 to now accommodate two sets of perceptions.

\section{Data}

$p_{i j} \quad$ interdictor's perception of the probability that the evader can traverse arc $(i, j)$ undetected when no sensor is installed

$q_{i j} \quad$ interdictor's perception of the probability that the evader can traverse $\operatorname{arc}(i, j)$ undetected when a sensor is installed; $q_{i j}<p_{i j}$

$p_{i j}^{2} \quad$ evader's perception of the probability that the evader can traverse $\operatorname{arc}(i, j)$ undetected when no sensor is installed

$q_{i j}^{2} \quad$ evader's perception of the probability that the evader can traverse arc $(i, j)$ undetected when a sensor is installed; $q_{i j}^{2} \leq p_{i j}^{2}$

\section{Evader's Decision Variables}

$y_{i j}, z_{i j} \quad$ analogous to $y_{i j}^{2}$ and $z_{i j}^{2}$ below, except that they are used to compute the interdictor's perception of the probability the evader avoids detection

$y_{i j}^{2} \quad$ positive only if evader traverses $(i, j)$ and no sensor is installed

$z_{i j}^{2} \quad$ positive only if evader traverses $(i, j)$ and a sensor is installed

\section{Boundary Conditions}

$$
x_{i j}, z_{i j}, z_{i j}^{2} \equiv 0 \quad(i, j) \notin A D
$$

In this setting, the interdictor knows both $\left(p_{i j}, q_{i j}\right)$ and $\left(p_{i j}^{2}, q_{i j}^{2}\right)$ for all arcs. While the interdictor views the latter as inferior estimates of these network parameters, the model implicitly assumes the interdictor knows all the information that the evader will use to select what the evader perceives to be a maximum-reliability $s^{\omega}-t^{\omega}$ path for all $\omega \in \Omega$.

PSNIP captures the case in which the evader is only aware of a subset of the sensor locations: If $p_{i j}^{2}>q_{i j}^{2}$ then the evader knows whether $(i, j)$ has a sensor, but if $p_{i j}^{2}=q_{i j}^{2}$ the evader is unaware that $(i, j)$ can receive a sensor. The case in which the evader is unaware of the sensors on all arcs (call it USNIP, for an uninformed evader) is easily handled because the evader's path selection has nothing to do with the interdictor's sensor-installation decisions. This is captured by a relatively straightforward modification of the SNIP model that we will not detail here. PSNIP generalizes SNIP in that the original model is recovered if $p_{i j}^{2}=p_{i j}$ and $q_{i j}^{2}=q_{i j}$ for all arcs, and also includes USNIP as a special case when $p_{i j}^{2}=q_{i j}^{2}=p_{i j}$ for all arcs.

PSNIP is formulated as SNIP (1), except that the definition of $h$ from (2) is replaced 
by

$$
\begin{array}{rlr}
h\left(x,\left(s^{\omega}, t^{\omega}\right)\right)=\max _{y, z, y^{2}, z^{2}} & y_{t^{\omega}} & \\
\text { s.t. } & (2 \mathrm{~b}),(2 \mathrm{c}),(2 \mathrm{~d}),(2 \mathrm{e}),(2 \mathrm{f}) & \\
& y_{i j} \leq M y_{i j}^{2}, & (i, j) \in A \\
& z_{i j} \leq M y_{i j}^{2}+M z_{i j}^{2}, & (i, j) \in A D \\
& \left(y^{2}, z^{2}\right) \in Y^{2}(x, \omega) . &
\end{array}
$$

Model (13) differs from (2) in that it has two sets of decision variables: the variables $\left(y^{2}, z^{2}\right)$ select the evader's path under $\left(s^{\omega}, t^{\omega}\right)$ and are constrained to be in $Y^{2}(x, \omega)$, which denotes the argmax of the evader's subproblem, i.e., (2) where $p$ and $q$ are replaced by the evader's perceptions $p^{2}$ and $q^{2}$. Variables $(y, z)$ are used for "accounting" purposes, i.e., to compute the interdictor's perception of the probability that the smuggler evades detection. Constraints (2e) and (13b) allow $y_{i j}$ to take positive flow only if $(i, j)$ has no sensor and $(i, j)$ is on the evader's optimal path. Similarly, $p_{i j}>q_{i j}$ and constraints (13c) allow $z_{i j}$ to be positive in an optimal solution to (13) only if $x_{i j}=1$ and either $y_{i j}^{2}>0$ or $z_{i j}^{2}>0$. We state the latter condition in this form because the evader may be unaware of a potential sensor $\left(p_{i j}^{2}=q_{i j}^{2}\right)$ and could traverse $(i, j)$ as if it has no sensor. Of course, in implementation we simply remove $z_{i j}^{2}$ and $q_{i j}^{2}$ from the smuggler's network but the above approach is notationally simpler.

It is typical that constraints of the form (13d) arise in a bilevel program. We avoided such constraints in SNIP because there the interdictor's and evader's objective functions were essentially identical, even though the former sought to minimize it and the latter sought to maximize it. Now, due to differing perceptions the objective functions differ.

The next task in the development is to achieve PSNIP's analog of (10), i.e., to reformulate PSNIP as a large-scale MIP. We only outline how to accomplish this and refer to [33] where this is carried out in detail. First, we state constraints (13d) in explicit form by: (i) writing the primal constraints of the evader's LP subproblem, i.e., constraints of (2) with $(p, q)$ replaced by $\left(p^{2}, q^{2}\right)$ and $(y, z)$ replaced by $\left(y^{2}, z^{2}\right)$, (ii) writing the dual constraints of this evader's LP subproblem, and (iii) enforcing strong duality of these primal and dual LPs. As a result, (13) can be stated as an LP (again, $x \in X$ is fixed in (13)). Second, to reformulate the resulting nested "min-max" problem, we use the kind of equivalent penaltybased reformulation employed in Section 2 and then take the dual of this inner maximizing LP for each $\omega \in \Omega$. The result is a single large-scale linear MIP for PSNIP defined on a general network. 
Instead of detailing the above process for PSNIP we turn to BiPSNIP, the special case of PSNIP defined on a bipartite network, and we carryout the type of steps outlined above to obtain a single large-scale MIP formulation for BiPSNIP. In Section 3, we denoted evader $\omega$ 's probability of traveling from $s^{\omega}$ to $t^{\omega}$ via checkpoint $k$ undetected as $\gamma_{k}^{\omega} p_{k}\left(\gamma_{k}^{\omega} q_{k}\right)$ without (with) a sensor installed at $k$, and for BiPSNIP these now denote the interdictor's perception of these probabilities. We add to this notation $\gamma_{k}^{2 \omega}, p_{k}^{2}$ and $q_{k}^{2}$, which represent the evader's perception. Let $\bar{q}^{2 \omega}=\max _{k \in A D^{\omega}} \gamma_{k}^{2 \omega} q_{k}^{2}$, and define $A D^{2 \omega}=\left\{k \in A D^{\omega}: \gamma_{k}^{2 \omega} p_{k}^{2} \geq \bar{q}^{2 \omega}\right\}$. Smuggler $\omega$ selects a checkpoint from $A D^{2 \omega}$ by solving the following model

$$
\begin{array}{ll}
\min _{\theta^{2 \omega}} & \theta^{2 \omega} \\
\text { s.t. } & \theta^{2 \omega} \geq r_{k}^{2 \omega}\left(1-x_{k}\right), k \in A D^{2 \omega}: v_{k},
\end{array}
$$

where $r_{k}^{2 \omega}=\gamma_{k}^{2 \omega} p_{k}^{2}-\bar{q}^{2 \omega}$ and where we have carried out the transformation to a problem with perfectly reliable detectors (from the smuggler's perspective) as done in Section 3 for BiSNIP. Decision variable $\theta^{2 \omega}$ plays the role that $\bar{\theta}^{\omega}$ played in (12), but here we suppress the "bar" notation for simplicity. The analog of constraints (13d) under scenario $\omega$ for BiPSNIP are represented by

$$
\begin{aligned}
\theta^{2 \omega} \geq r_{k}^{2 \omega}\left(1-x_{k}\right), \quad k \in A D^{2 \omega} \\
\sum_{k \in A D^{2 \omega}} v_{k}^{\omega}=1, \quad v_{k}^{\omega} \geq 0, \quad k \in A D^{2 \omega} \\
\theta^{2 \omega}=\sum_{k \in A D^{2 \omega}} r_{k}^{2 \omega}\left(1-x_{k}\right) v_{k}^{\omega} .
\end{aligned}
$$

Here, the optimality conditions for (14) are represented by primal feasibility (15a), dual feasibility (15b), and strong duality (15c). For fixed $x \in X,(15)$ is a linear system. We assume that (15) has a unique solution for each $x \in X$, i.e., that the smuggler is not indifferent between selecting two or more checkpoints. Note that $v_{k}^{\omega}, k \in A D^{2 \omega}$, takes value one for the checkpoint selected by smuggler $\omega$ and is zero otherwise.

We now represent BiPSNIP as

$$
\begin{array}{rll}
\min _{x, \theta, \theta^{2}, v} & \sum_{\omega \in \Omega} p^{\omega} \theta^{\omega} & \\
\text { s.t. } & x \in X & \\
& \theta^{\omega} \geq \gamma_{k}^{\omega} p_{k}\left(1-x_{k}\right) v_{k}^{\omega}, & k \in A D^{2 \omega}, \omega \in \Omega \\
& \theta^{\omega} \geq \gamma_{k}^{\omega} q_{k} x_{k} v_{k}^{\omega}, & k \in A D^{2 \omega}, \omega \in \Omega \\
& (15 \mathrm{a}),(15 \mathrm{~b}),(15 \mathrm{c}), \quad \omega \in \Omega .
\end{array}
$$


BiPSNIP (16) differs from BiSNIP (11) in that for each $\omega,(16 \mathrm{a})$ and (16b) are now enforced only at the checkpoint $k$ that evader $\omega$ selects via $v_{k}^{\omega}$. (In (11) this is not necessary since the identical-perceptions assumption leads to (11a) and (11b) being tight at the maximum of their right-hand-side values for each $\omega$.) Optimality of $v_{k}^{\omega}$ with respect to the evader's perception is ensured by (15a)-(15c). As stated, (16) is a nonlinear MIP due to the product of the binary variables $x_{k}$ and $v_{k}^{\omega}$. This is easily linearized as captured in the following linear MIP:

$$
\begin{aligned}
& \min _{x, \theta, \theta^{2}, v, y, z} \sum_{\omega \in \Omega} p^{\omega} \theta^{\omega} \\
& \text { s.t. } \quad x \in X \\
& \theta^{\omega} \geq \gamma_{k}^{\omega} p_{k} y_{k}^{\omega}, \quad k \in A D^{2 \omega}, \omega \in \Omega \\
& \theta^{\omega} \geq \gamma_{k}^{\omega} q_{k} z_{k}^{\omega}, \quad k \in A D^{2 \omega}, \omega \in \Omega \\
& \theta^{2 \omega} \geq r_{k}^{2 \omega}\left(1-x_{k}\right), \quad k \in A D^{2 \omega}, \omega \in \Omega \\
& \sum_{k \in A D^{2 \omega}} v_{k}^{\omega}=1, \quad v_{k}^{\omega} \geq 0, \quad k \in A D^{2 \omega}, \omega \in \Omega \\
& \theta^{2 \omega}=\sum_{k \in A D^{2 \omega}} r_{k}^{2 \omega} y_{k}^{\omega}, \quad \omega \in \Omega \\
& y_{k}^{\omega} \leq v_{k}^{\omega}, \quad k \in A D^{2 \omega}, \omega \in \Omega \\
& 0 \leq y_{k}^{\omega} \leq 1-x_{k}, \quad k \in A D^{2 \omega}, \omega \in \Omega \\
& z_{k}^{\omega} \geq v_{k}^{\omega}+x_{k}-1, \quad k \in A D^{2 \omega}, \omega \in \Omega .
\end{aligned}
$$

Constraints (17a) and (17b) are equivalent to (16a) and (16b) under the linearization of $\left(1-x_{k}\right) v_{k}^{\omega}$ by $y_{k}^{\omega}$ via (17f) and (17g) and the linearization of $x_{k} v_{k}^{\omega}$ by $z_{k}^{\omega}$ in (17h). The optimality conditions from (15a)-(15c) for the smuggler's behavior are captured in (17c)(17e) and again include the linearization of $\left(1-x_{k}\right) v_{k}^{\omega}$ by $y_{k}^{\omega}$.

We have assumed the evader's response in BiPSNIP is unique for each $x \in X$, and in this case there is no ambiguity in how the evader's behavior is being modeled. However, consider (17) when this is not the case. Due to the simultaneous minimization over all the decision variables with respect to the interdictor's objective function, among the optimal responses of the evader (separately, for each $\omega \in \Omega$ ), the decision most beneficial to the interdictor is selected. So, when indifferent with respect to two paths (under the evader's perception) the evader effectively cooperates with the interdictor. Now, this might happen assuming the smuggler is unaware of the interdictor's perception, but even in this case it is clearly an optimistic assumption from the interdictor's point of view. An alternative formulation which involves introducing a nested " $\min _{x, \theta} \max _{\theta^{2}, v, y, z}$ " could be formulated to 
yield the corresponding uncooperative or pessimistic solution, but we do not pursue this here. This issue is not specific to the interdiction setting, and such methods for dealing with non-unique responses have been investigated in the bilevel programming literature [6].

\section{Step Inequalities for BiSNIP}

Our initial attempts to solve BiSNIP (12) using branch-and-bound (B\&B) codes indicated that BiSNIP's LP relaxation can be loose. Hence, we seek to tighten the formulation with a class of valid inequalities that we term step inequalities. In this section, we develop the inequalities and explain them from an intuitive perspective. Then, we describe a separation procedure that, given a solution to an LP relaxation of BiSNIP, can efficiently identify a most violated step inequality or prove that there are no violated inequalities. We report our computational experience on iteratively generating step inequalities using this separation procedure to help solve BiSNIP.

Consider BiSNIP (12), and let $T(\omega)=\left\{k_{1}, k_{2}, \cdots, k_{\ell}\right\} \subseteq A D^{\omega}$ satisfy

$$
r_{k_{1}}^{\omega} \geq r_{k_{2}}^{\omega} \geq \cdots \geq r_{k_{\ell}}^{\omega}>0
$$

We define a step inequality on $T(\omega)$ as

$$
\bar{\theta}^{\omega} \geq r_{k_{1}}^{\omega}-\left(r_{k_{1}}^{\omega}-r_{k_{2}}^{\omega}\right) x_{k_{1}}-\cdots-\left(r_{k_{\ell}}^{\omega}-0\right) x_{k_{\ell}}
$$

When $\ell=1$, the one-step inequality (19) is simply an existing constraint in (12). In general, when $\ell \geq 2$ the step inequalities are not redundant, at least when $x$ takes on fractional values in the convex hull of $X$. Consider a two-step inequality, i.e., (19) with $\ell=2: \bar{\theta}^{\omega} \geq r_{k_{1}}^{\omega}-\left(r_{k_{1}}^{\omega}-r_{k_{2}}^{\omega}\right) x_{k_{1}}-\left(r_{k_{2}}^{\omega}-0\right) x_{k_{2}}$. If $x_{k_{1}}=x_{k_{2}}=0$ then the smuggler

will select $k_{1}$ and $\bar{\theta}^{\omega}=r_{k_{1}}^{\omega}$ by the two-step inequality. If $x_{k_{1}}=1$ then the two $r_{k_{1}}^{\omega}$ terms in the step inequality cancel and $\bar{\theta}^{\omega}$ "steps down" to the residual term $r_{k_{2}}^{\omega}\left(1-x_{k_{2}}\right)$ so that the two-step inequality collapses to a one-step inequality, i.e., an existing constraint in (12). More generally, the step inequality exploits the ordering among different evasion paths and effectively reduces the maximal evasion probability one step a time as further sensors are installed. Of course, the value of the step inequality is that it removes fractional solutions that are otherwise feasible to the LP relaxation of BiSNIP. This can be viewed by continuously increasing $x_{k_{1}}$ from 0 to 1 and then continuously increasing $x_{k_{2}}$ from 0 to 1 and so on.

There are an exponential number of step inequalities, and adding all possible step inequalities to BiSNIP is out of the question. So, we instead iteratively solve the linear 
programming relaxation of BiSNIP and add step inequalities on an as-needed basis. This procedure is repeated until no violated step inequalities remain. The separation problem for step inequalities requires that given $\left(x^{l p}, \bar{\theta}^{l p}\right)$, a feasible solution to the LP relaxation of (12), we either identify a most violated step inequality for each $\omega$ or determine that none are violated. We restrict the set $T(\omega)$ defining the step inequality to include $k_{1}$, where $r_{k_{1}}^{\omega}=\max _{k \in A D^{\omega}} r_{k}^{\omega}$, and to satisfy the ordering condition (18). Then, to maximize the right-hand side of (19) over such $T(\omega) \subset A D^{\omega}$ we solve

$$
\nu^{\omega}=\min _{T(\omega) \subset A D^{\omega}} \sum_{k_{i} \in T(\omega)}\left(r_{k_{i}}^{\omega}-r_{k_{i+1}}^{\omega}\right) x_{k_{i}}^{l p}
$$

for each $\omega \in \Omega$, where $r_{k_{\ell+1}}^{\omega} \equiv 0$. We solve (20) efficiently by solving a shortest-path problem on an acyclic network $G(V, E)$ in which $V=A D^{\omega} \cup\left\{k_{\left|A D^{\omega}\right|+1}\right\}$ with $r_{k_{\left|A D^{\omega}\right|+1}^{\omega}}^{\omega} \equiv 0$. The edge set $E$ contains a directed arc from node $k_{i}$ to $k_{j}$ only if $r_{k_{i}}^{\omega} \leq r_{k_{j}}^{\omega}$; the associated arc length is $\left(r_{k_{j}}^{\omega}-r_{k_{i}}^{\omega}\right) x_{k_{j}}^{l p}$. We solve the shortest path problem from node $k_{\left|A D^{\omega}\right|+1}$ to node $k_{1}$ over $G(V, E)$ and obtain optimal value $\nu^{\omega}$. The nodes from $V$ on a shortest path define $T^{*}(\omega)$ solving (20). If $\bar{\theta}^{\omega, l p} \geq r_{k_{1}}^{\omega}-\nu^{\omega}$ then there are no violated step inequalities for $\omega$ at $\left(x^{l p}, \bar{\theta}^{l p}\right)$. Otherwise, the checkpoints $T^{*}(\omega)$ define a most violated step inequality for $\omega$.

\begin{tabular}{l|rr|rrrr} 
& \multicolumn{2}{|c|}{ CPLEx B\&B } & \multicolumn{4}{|c}{ With Step Inequalities } \\
\hline$b$ & rel. gap. (\%) & comp. time & rel. gap. (\%) & comp. time & no. of $\geq$ & iters. \\
\hline 30 & 20.2 & 489 & 0.02 & 8 & 465 & 5 \\
40 & 20.9 & 559 & 0.00 & 6 & 405 & 4 \\
50 & 22.8 & 1913 & 0.00 & 11 & 657 & 8 \\
60 & 25.7 & 7779 & 0.33 & 17 & 651 & 17 \\
70 & 27.6 & 35428 & 1.02 & 98 & 639 & 7 \\
80 & 27.7 & 13251 & 0.00 & 10 & 489 & 6 \\
90 & 28.7 & 10130 & 0.28 & 15 & 530 & 5 \\
100 & 29.0 & 10923 & 0.34 & 16 & 522 & 6 \\
110 & 28.0 & 3215 & 0.19 & 13 & 594 & 8 \\
120 & 26.4 & 256 & 0.45 & 14 & 419 & 8
\end{tabular}

Table 1: Computational results for: (i) solving (12) directly using CpLEx and (ii) iteratively adding violated step inequalities to the initial LP relaxation and then proceeding with B\&B. "rel. gap (\%)" is $100 \cdot\left(z_{I P}-z_{L P}\right) / z_{L P}$, "comp. time" reports total computation time in seconds, "no. of $\geq$ " reports the number of step inequalities generated and "iters." reports the total number of major iterations.

Table 1 shows our computational results for a test problem with 85 origins, 263 customs checkpoints, 9 destinations, and $|\Omega|=306$ scenarios. We assume $c_{i j}=1$ for all $(i, j) \in A D$, so that the budget constraint is simply a cardinality constraint, and we solve our test problem for various values of the budget $b$ on a $1.7 \mathrm{GHz}$, Dell Xeon dual-processor machine 
with 2 GB of memory. In our test problem, the average cardinality of $A D^{\omega}$ is about 20 . The separation procedure for the step inequalities was coded in $\mathrm{C}++$ and the MIP problems were solved with the CPLEx Concert Technology libraries (version 9.0).

All MIPs were solved with a relative tolerance of $0.1 \%$, i.e., the B\&B algorithm was terminated when $100 \cdot(\bar{z}-\underline{z}) / \underline{z} \leq 0.1$. Here, $\bar{z}$ is the objective function value of the B\&B algorithm's current incumbent solution, and $\underline{z}$ is B\&B's current lower bound on the MIP's optimal value. We use $z_{I P}$ to denote $\bar{z}$ when B\&B terminates, and we use $z_{L P}$ to denote the optimal value of the initial LP relaxation, i.e., $\underline{z}$ at the beginning of B\&B. In Table 1, the $z_{L P}$ values used to compute the "rel. gap" are those of (12)'s LP relaxation under the "CPLEx B\&B" heading and those of the same LP relaxation after the addition of the step inequalities under the "With Step Inequalities" heading. We iteratively added step inequalities in a sequence of "major iterations" until the maximum violation was less than $10^{-6}$. The computation times reported under "With Step Inequalities" include the time to generate the step inequalities and the time to solve the resulting MIPs. As Table 1 shows, the use of step inequalities can considerably tighten the optimal value of the initial LP relaxation, and our results suggest that in turn, this can significantly reduce required computational effort, particularly on the most challenging instances.

We close this section by noting that in [33], the step inequalities described here have been extended, with computational success, to the SNIP model on a general network. However, to do so first requires detailed development of an L-Shaped decomposition method for SNIP, which is beyond the scope of this paper.

\section{Conclusion}

We have described two types of stochastic network interdiction models whose solution can be used to select sites to install sensors for detecting smuggled nuclear material. This work is motivated by the US Department of Energy's Second Line of Defense Program. In both models, our goal is to minimize the probability that a smuggler can successfully travel through an underlying transportation network undetected. Our two models differ with respect to whether the interdictor and smuggler have the same (SNIP) or differing (PSNIP) perceptions of the detection probabilities on the network's arcs. In SNIP, the smuggler is aware of the locations of all sensors that have been installed. PSNIP can capture the case in which the smuggler is aware of only a subset of the sensor locations. For both SNIP and PSNIP we developed the special case of the model in which sensors can only be installed at 
border crossings of a single country, namely Russia. Our experience with the former special case, BiSNIP, indicates significant computational benefit from using a new class of valid inequalities called step inequalities.

\section{Acknowledgements}

This research was partially supported by the National Science Foundation under Grants DMI-0217927 and DMI-0228419. The authors also thank Steven M. Dinehart of the Los Alamos National Laboratory SLD team and Bruce Pentola of the Department of Energy's National Nuclear Security Administration (NA-265). We are grateful to three anonymous referees and an Associate Editor whose suggestions improved the paper.

\section{References}

[1] R.K. Ahuja, T.L. Magnanti, and J.B. Orlin. Network Flows. Prentice Hall, Upper Saddle River, NJ, 1993.

[2] M.D. Bailey and H. Bayrak. Network interdiction under asymmetric information. Technical report, Industrial Engineering Department, University of Pittsburgh, 2005.

[3] M.D. Bailey, S.M. Shechter, and A.J. Schaefer. SPAR: stochastic programming with adversarial recourse. Technical report, Industrial Engineering Department, University of Pittsburgh, 2004.

[4] D.Y. Ball. The US Second Line of Defense: Preventing nuclear smuggling across Russia's borders. Technical report, Center for the Strategic and International Studies, Washington, DC, 1998. PONARS Memo \# 50.

[5] M.O. Ball, B.L. Golden, and R.V. Vohra. Finding the most vital arcs in a network. Operations Research Letters, 8:73-76, 1989.

[6] J.F. Bard. Some properties of the bilevel programming problem. Journal of Optimization Theory and Applications, 68:371-378, 1991.

[7] J.F. Bard. Practical Bilevel Optimization: Algorithms and Applications. Kluwer Academic Publishers, Boston, MA, 1998.

[8] O. Ben-Ayed. Bi-level linear programming. Computers and Operations Research, 20:485-501, 1993.

[9] J.F. Benders. Partitioning procedures for solving mixed-variables programming problems. Numerische Mathematik, 4:238-252, 1962.

[10] G.G. Brown, M. Carlyle, R. Harney, E. Skroch, and R.K. Wood. How to optimally interdict a belligerent project to develop a nuclear weapon. Technical report, Operations Research Department, Naval Postgraduate School, 2004. 
[11] M.S. Chern and K.C. Lin. Interdicting the activities of a linear program - a parametric approach. European Journal of Operational Research, 86:580-591, 1995.

[12] D. Cobb. Statement of Associate Director, Threat Reduction, Los Alamos National Laboratory, submitted to Committee on Energy and Natural Resources, US Senate, July 10, 2002.

[13] H.W. Corley and D.Y. Sha. Most vital links and nodes in weighted networks. Operations Research Letters, 1:157-160, 1982.

[14] K. Cormican, D.P. Morton, and R.K. Wood. Stochastic network interdiction. Operations Research, 46:184-197, 1998.

[15] Department of Energy Press Release: Bill Richardson, Russian Federation Dedicate "Second Line of Defense", US Nuclear Detection Technology to Help Secure Russian Borders, September 2, 1998.

[16] Department of Energy and National Nuclear Security Administration, Overview of NA-25: Office of International Material Protection and Cooperation. http://www.nnsa.doe.gov/na-20/na25_fact.shtml.

[17] D.R. Fulkerson and G.C. Harding. Maximizing the minimum source-sink path subject to a budget constraint. Mathematical Programming, 13:116-118, 1977.

[18] General Accounting Office, Nuclear Nonproliferation: US Efforts to Help Other Countries Combat Nuclear Smuggling Need Strengthened Coordination and Planning, Report to the Ranking Minority Member, Subcommittee on Emerging Threats, and Capabilities, Committee on Armed Services, US Senate, GAO-02-426, May 2002.

[19] A.M. Geoffrion. Generalized Benders decomposition. Journal of Optimization Theory and Applications, 10:237-260, 1972.

[20] P.M. Ghare, D.C. Montgomery, and T.M. Turner. Optimal interdiction policy for a flow network. Naval Research Logistics Quarterly, 18:37-45, 1971.

[21] B. Golden. A problem in network interdiction. Naval Research Logistics Quarterly, 25:711-713, 1978.

[22] R. Hemmecke, R. Schultz, and D.L. Woodruff. Interdicting stochastic networks with binary interdiction effort. In D.L. Woodruff, editor, Network Interdiction and Stochastic Integer Programming. Kluwer Academic Publishers, Boston, MA, 2003.

[23] International Atomic Energy Agency, Illicit Nuclear Trafficking Database. http://www.iaea.org/NewsCenter/Features/RadSources/Fact_Figures.html.

[24] E. Israeli and R.K. Wood. System interdiction and defense. Working paper, Operations Research Department, Naval Postgraduate School, 2001.

[25] E. Israeli and R.K. Wood. Shortest-path network interdiction. Networks, 40:97-111, 2002 . 
[26] Y. Ishizuka K. Shimizu and J.F. Bard. Nondifferentiable and Two-Level Programming. Kluwer Academic Publishers, Boston, MA, 1997.

[27] J.E. Kelley. The cutting plane method for solving convex programs. SIAM Journal of Industrial and Applied Mathematics, 8:703-712, 1960.

[28] G. Laporte and F.V. Louveaux. The integer $L$-shaped method for stochastic integer programs with complete recourse. Operations Research Letters, 13:133-142, 1993.

[29] K. Malik, A.K. Mittal, and S.K. Gupta. The k-most vital arcs in the shortest path problem. Operations Research Letters, 8:223-227, 1989.

[30] A.W. McMasters and T.M. Mustin. Optimal interdiction of a supply network. Naval Research Logistics Quarterly, 17:261-268, 1970.

[31] D.P. Morton and R.K. Wood. Restricted-recourse bounds for stochastic linear programming. Operations Research, 47:943-956, 1999.

[32] Nuclear Threat Initiative, Interdicting Nuclear Smuggling, Second Line of Defense. http://www.nti.org/e_research/cnwm/interdicting/second.asp.

[33] F. Pan. Models and Methods for Stochastic Network Interdiction. PhD thesis, The University of Texas at Austin, 2005.

[34] F. Pan, W. Charlton, and D.P. Morton. Interdicting smuggled nuclear material. In D.L. Woodruff, editor, Network Interdiction and Stochastic Integer Programming. Kluwer Academic Publishers, Boston, MA, 2003.

[35] B.K. Reed. Models for Proliferation Interdiction Response Analysis. MS thesis, Naval Postgraduate School, 1994.

[36] R.M. Van Slyke and R.J.-B. Wets. L-shaped linear programs with applications to optimal control and stochastic programming. SIAM Journal on Applied Mathematics, 17:638-663, 1969.

[37] A.R. Washburn and R.K. Wood. Two-person zero-sum games for network interdiction. Operations Research, 43:243-251, 1994.

[38] R.D. Wollmer. Removing arcs from a network. Operations Research, 12:934-940, 1964.

[39] R.D. Wollmer. Two-stage linear programming under uncertainty with 0-1 integer first stage variables. Mathematical Programming, 19:279-288, 1980.

[40] R.K. Wood. Deterministic network interdiction. Mathematical and Computer Modeling, 17:1-18, 1993. 